\title{
The TCA benchmark for validation of temperature feedback calculations
}

\author{
Steven C. van der Marck, Nicola L. Asquith \\ Nuclear Research and consultancy Group (NRG) \\ P.O. Box 25, 1755 ZG Petten, Netherlands \\ vandermarck@nrg.eu, asquith@nrg.eu
}

\begin{abstract}
The TCA benchmark was investigated as a possible candidate for validation of temperature feedback calculations. This benchmark has low-enriched uranium fuel, light water moderation and reflection, and a temperature range of $20-60{ }^{\circ} \mathrm{C}$. The use of three different nuclear data libraries was considered, viz. ENDF/B-VIII.0, JEFF-3.3, and JENDL-4.0. Since the results were not as good as hoped for, additional studies were performed to identify the cause(s) of discrepancies.

The benchmark values depend on a choice of delayed neutron data, so it was investigated whether this could be the cause of discrepancies. Also, an assessment was made based on critical configurations only, i.e. avoiding the use of delayed neutron data, whether the calculations exhibit a bias in relation to the benchmark in the results for the effect of temperature. Indications were found that such a bias exists.

It is concluded that the choice of delayed neutron data has a significant effect on the benchmark values themselves. The use of three major nuclear data libraries leads to a range of benchmark values for each configuration, rather than one value. Also, one has to take into account the possibility of a bias in the calculation of temperature effects. It is not clear at this point what the cause of the bias is.
\end{abstract}

KEYWORDS: temperature feedback, reactivity, TCA benchmark

\section{INTRODUCTION}

The effect that temperature has on the reactivity of a reactor is important. A negative temperature feedback can be an important safety feature. Calculations of temperature effects therefore merit careful attention and need to be validated. The International Reactor Physics Experiments Handbook (IRPhE) [1] provides several benchmarks that can be used for this purpose. For the present paper the TCA benchmark was selected, because it has many features in common with the High Flux Reactor in Petten.

Benchmark calculations were performed for TCA, as reported in Section 2. The results were not entirely convincing, prompting further study. One topic was the role of delayed neutron data in the benchmark. In Section 3 it is argued that the benchmark values published in Ref. [1] involve an interpretation based on a specific delayed neutron data set. In Section 3 the benchmark values are re-evaluated used different nuclear data, leading to a different comparison between calculations and benchmark values, but still not a convincing one. It was investigated whether, by sidestepping the role of delayed nuclear data, one can establish whether there is a bias between calculations and benchmark, see Section 4. The conclusions are presented in Section 5. 


\section{CALCULATIONS FOR THE TCA BENCHMARK "AS IS"}

\subsection{Short Benchmark Description}

Experiments were carried out in the Tank-type Critical Assembly (TCA) in Japan Atomic energy Research Institute (JAERI) in 1988-1989. The core configurations consisted of low enriched $\mathrm{UO}_{2}$ rods, moderated and reflected by light water. There were experiments with water without poisons (A-cores), ones with $\mathrm{H}_{3} \mathrm{BO}_{3}$ (B-cores), and ones with $\mathrm{Gd}\left(\mathrm{NO}_{3}\right)_{3}$ (C-cores).

For each of the configurations, several different temperatures were realized, and the water level was adjusted to reach a critical state. The critical water height is given in the benchmark description. For the $\mathrm{A}$ - and B-cores four different temperatures were realized, and for the $\mathrm{C}$-cores five different temperatures.

Subsequently the water height was slightly increased above the critical level in order to obtain the differential reactivity worth of the water level by the positive reactor period method. The measured quantities were the water height increase $(\Delta H)$ and the reactor period $(\tau)$. Based on the measured values for these quantities, the equation

$$
\Delta \rho(\tau)=\frac{1}{\beta} \sum_{n=U 235, U 238} \sum_{i=1}^{6} \frac{\beta_{i, n}}{1+\lambda_{i, n} \tau}
$$

was used to get a value for the reactivity in units of a dollar (\$). In this equation $\beta_{i, n}$ is the effective delayed neutron fraction of nuclide $n$ in the $i^{\text {th }}$ time group, $\beta$ is the total effective delayed neutron fraction of nuclide $n$, and $\lambda_{i, n}$ is the time constant of the $i^{\text {th }}$ time group of nuclide $n$. In this equation it is assumed that the number of time groups is 6 .

\subsection{Codes and Data}

Calculations were performed using MCNP version 6.1.1 [2]. Models for the various configurations of TCA were developed from scratch, solely on the basis of the IRPhE benchmark description. The temperature in each (non-void) cell was set according to the benchmark specification. Since the nuclear data were also processed for exactly the same temperature, this means that no temperature treatment is performed by MCNP. The MCNP option 'kopts' was used for calculating reactor kinetics parameters $\beta$ and $\lambda$, using a block size of 10 .

Three different nuclear data libraries were used, viz. ENDF/B-VIII.0 [3], JEFF-3.3 [4], and JENDL-4.0 [5]. The nuclides in the benchmark, ${ }^{1} \mathrm{H},{ }^{10,11} \mathrm{~B},{ }^{14} \mathrm{~N},{ }^{16} \mathrm{O},{ }^{27} \mathrm{Al},{ }^{152,154,155,156,157,158,160} \mathrm{Gd},{ }^{234,235,238} \mathrm{U}$ were processed using NJOY-2016 [6]. The processing was performed using the same NJOY input as for the criticality safety benchmarking in Refs. [3] and [4]. This processing was repeated for each of the temperatures in the TCA benchmark.

The thermal scattering data had to be treated differently. Using NJOY one can only process thermal scattering data at temperatures specified in the ENDF evaluation. To create thermal scattering ACE files for the exact temperatures used in the TCA benchmark, the MCNP utility MAKXSF was used to interpolate between NJOY generated ACE files. It was checked with the new interpolation method of Marquez Damian [7] that the MAKXSF interpolation did not introduce a bias in the calculated values for the temperature coefficient of reactivity. 


\subsection{Fixed Water Height at Several Temperatures}

For each of the TCA configurations, the water height was fixed at a certain level. Using this water level, calculations were performed using nuclear data that were processed for the exact temperature of the subcase at hand. The fixed water level $\left(H_{c}\right)$ per case was calculated using the fit in the benchmark description:

$$
H_{c}(T)=a_{0}+a_{1} T+a_{2} T^{2}+a_{3} T^{3} .
$$

The values for the fit parameters $a_{0}-a_{3}$ were copied from the benchmark description. For each benchmark case the fixed water level was set to $H_{c}\left(T=40{ }^{\circ} \mathrm{C}\right)$. The values are listed in Table I.

Table I. Water height: for each of the configurations, the water height was fixed in the calculations, at the level calculated by using Eq. (2).

\begin{tabular}{cc|cc|cc}
\hline Case & $\begin{array}{c}H_{c}\left(\mathrm{~T}=40^{\circ} \mathrm{C}\right) \\
(\mathrm{cm})\end{array}$ & Case & $\begin{array}{c}H_{c}\left(\mathrm{~T}=40{ }^{\circ} \mathrm{C}\right) \\
(\mathrm{cm})\end{array}$ & Case & $\begin{array}{c}H_{c}\left(\mathrm{~T}=40^{\circ} \mathrm{C}\right) \\
(\mathrm{cm})\end{array}$ \\
\hline A1a & 46.4627 & $\mathrm{~B} 1$ & 52.5556 & $\mathrm{C} 1$ & 83.7036 \\
A2a & 60.9570 & $\mathrm{~B} 2$ & 60.9000 & $\mathrm{C} 2$ & 91.7717 \\
A3 & 91.9521 & B3 & 66.7590 & $\mathrm{C} 3$ & 96.0923 \\
\hline
\end{tabular}

\subsection{From k-Eigenvalues to Temperature Feedback Coefficient}

The values for $k_{\text {eff }}$ were translated into values for reactivity $\rho$, using $\rho=1-1 / k_{\text {eff }}$. The values at different temperatures were fitted to the polynomial

$$
\rho(T)=r_{0}+r_{1} T+r_{2} T^{2}+r_{3} T^{3} .
$$

The temperature feedback $\alpha(T)$ was calculated as the derivative of $\rho(T)$ :

$$
\alpha(T)=\frac{d \rho(T)}{d T}=r_{1}+2 r_{2} T+3 r_{3} T^{2} .
$$

The benchmark values for the feedback coefficient are given in units of 'dollar cents' per degree. Therefore the value obtained here for $\alpha\left(T=40{ }^{\circ} \mathrm{C}\right)$ was divided by the effective delayed neutron fraction $\beta$. The value for $\beta$ given in the benchmark description was used.

\subsection{Results}

The calculated results for the temperature feedback coefficient are reasonably close to the benchmark values. The results for the first two pure water cases compare favorably with the benchmark value, when taking uncertainties into account. This does not hold for the smallest configuration with pure water, A3, for which the calculated value is significantly higher than the benchmark value for all three nuclear data libraries.

The results for the borated water cases are all reasonably close to the benchmark value when taking the uncertainties into account. The results for the cases with gadolinium in the water show a bias: all results are higher than the benchmark values, for all three nuclear data libraries. In general one can say, however, 
that the differences between the benchmark values and the results based on the various libraries are not large.

Table II. Results: calculated values for the temperature coefficient of reactivity, using three different nuclear data libraries, compared with the benchmark value. The units are $(\$ / 100) /{ }^{\circ} \mathrm{C}$.

\begin{tabular}{ccccc}
\hline & Benchmark & ENDF/B-VIII.0 & JEFF-3.3 & JENDL-4.0 \\
\hline A1a & $-1.64 \pm 0.18$ & $-1.44 \pm 0.08$ & $-1.40 \pm 0.07$ & $-1.46 \pm 0.08$ \\
A2a & $-1.24 \pm 0.10$ & $-1.20 \pm 0.08$ & $-1.22 \pm 0.07$ & $-1.21 \pm 0.07$ \\
A3 & $-1.20 \pm 0.05$ & $-0.98 \pm 0.05$ & $-0.87 \pm 0.04$ & $-1.00 \pm 0.05$ \\
\hline B1 & $-1.11 \pm 0.28$ & $-1.28 \pm 0.10$ & $-1.13 \pm 0.10$ & $-1.46 \pm 0.10$ \\
B2 & $-1.27 \pm 0.11$ & $-1.24 \pm 0.08$ & $-1.07 \pm 0.08$ & $-1.21 \pm 0.08$ \\
B3 & $-1.07 \pm 0.09$ & $-0.88 \pm 0.08$ & $-0.96 \pm 0.08$ & $-1.08 \pm 0.08$ \\
\hline C1 & $-0.80 \pm 0.07$ & $-0.53 \pm 0.04$ & $-0.51 \pm 0.05$ & $-0.69 \pm 0.04$ \\
C2 & $-0.59 \pm 0.08$ & $-0.26 \pm 0.04$ & $-0.10 \pm 0.05$ & $-0.32 \pm 0.05$ \\
C3 & $+0.16 \pm 0.08$ & $+0.43 \pm 0.04$ & $+0.49 \pm 0.05$ & $+0.48 \pm 0.05$ \\
\hline
\end{tabular}

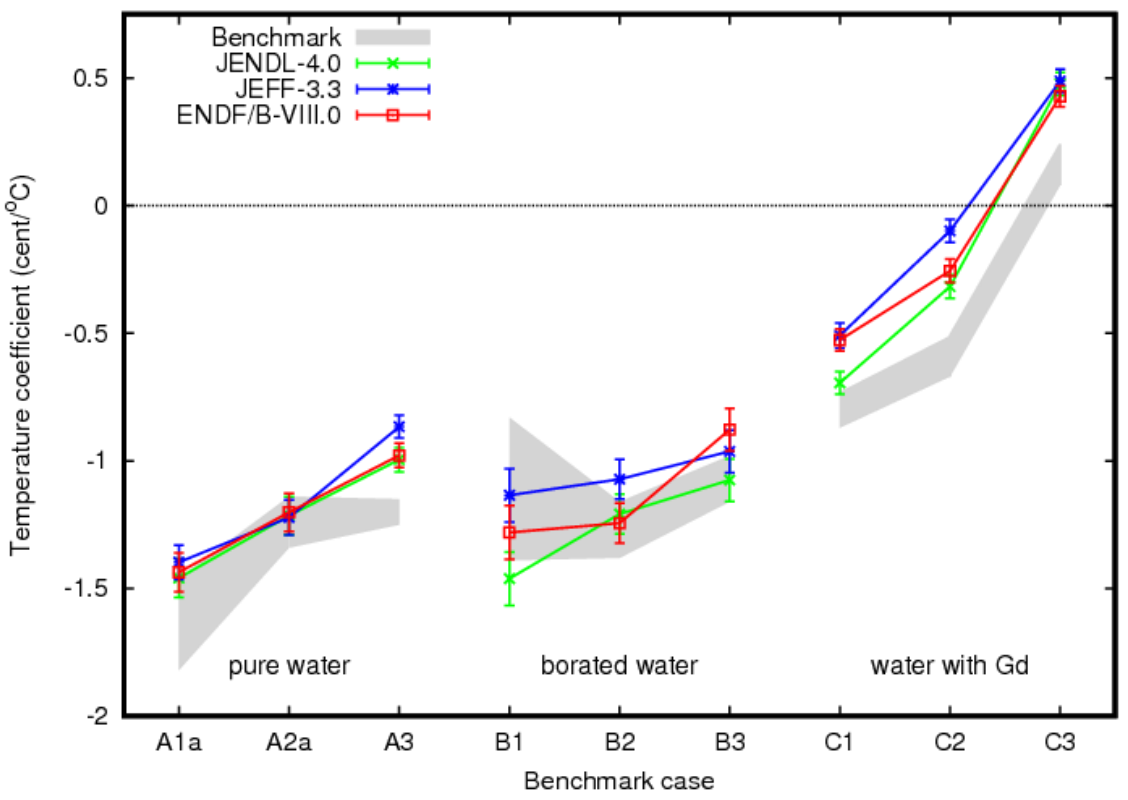

Figure 1. Results: calculated values for the temperature coefficient of reactivity, using three different nuclear data libraries, compared with the benchmark value.

It should be noted that the analysis so far makes inconsistent use of nuclear data. The benchmark value in IRPhE is based on an analysis of the raw data, making use of calculated values for the effective delayed neutron fraction (in six time groups) and the time constants (in the same time groups). These calculations were performed by the benchmark evaluators, using the code DANTSYS with nuclear data from JENDL3.3. For the calculations presented here, a different code and different nuclear data libraries were used. Also for the calculated temperature coefficients it was necessary to convert the unit of reactivity to dollar- 
cents. This was done by dividing by the value for $\beta$ calculated with DANTSYS with JENDL-3.3 nuclear data.

\section{CONSISTENT USE OF NUCLEAR DATA}

This use of different sets of nuclear data for one and the same benchmark calculation is not necessary. The MCNP models for the various configurations can be used to calculate $\beta$ and $\lambda$. This was done for each of the four (A and B) or five (C) configurations per benchmark case, each case with its water height as specified in the benchmark description. The averaged results for $\beta$ are shown in Table III. In some cases there are non-negligible differences with the values from the IRPhE documentation. For brevity the results for the partial $\beta$ 's are not listed here.

The values for the time constants as defined in the various nuclear data libraries are listed in Table IV. The values used in the IRPhE benchmark description are the same as the JENDL-4.0 values rounded off to three significant digits (in fact, these are the values proposed in 1965 by Keepin). The values in ENDF/BVIII.0 and JEFF-3.3 are rather different. The data in JEFF-3.3 are divided into eight time groups, as proposed in Ref. [8], making it impossible to compare the results per group with other libraries.

Table III. Effective delayed neutron fraction: results using the various nuclear data libraries compared with the values listed in the IRPhE benchmark description.

\begin{tabular}{cccccccc}
\hline & IRPhE & \multicolumn{2}{c}{ ENDF/B-VIII.0 } & \multicolumn{2}{c}{ JEFF-3.3 } & \multicolumn{2}{c}{ JENDL-4.0 } \\
& $\beta(\mathrm{pcm})$ & $\beta(\mathrm{pcm})$ & Difference & $\beta(\mathrm{pcm})$ & Difference & $\beta(\mathrm{pcm})$ & Difference \\
\hline A1a & 753.22 & $772 \pm 6$ & $(2.5 \pm 0.8) \%$ & $802 \pm 6$ & $(6.5 \pm 0.7) \%$ & $782 \pm 6$ & $(3.8 \pm 0.8) \%$ \\
$\mathrm{~A} 2 \mathrm{a}$ & 759.37 & $778 \pm 6$ & $(2.5 \pm 0.8) \%$ & $796 \pm 6$ & $(4.8 \pm 0.8) \%$ & $782 \pm 6$ & $(3.0 \pm 0.8) \%$ \\
$\mathrm{~A} 3$ & 764.45 & $770 \pm 6$ & $(0.7 \pm 0.8) \%$ & $796 \pm 6$ & $(4.1 \pm 0.8) \%$ & $775 \pm 6$ & $(1.4 \pm 0.8) \%$ \\
$\mathrm{~B} 1$ & 754.04 & $761 \pm 6$ & $(0.9 \pm 0.8) \%$ & $790 \pm 6$ & $(4.8 \pm 0.8) \%$ & $774 \pm 6$ & $(2.6 \pm 0.8) \%$ \\
$\mathrm{~B} 2$ & 754.17 & $765 \pm 6$ & $(1.4 \pm 0.8) \%$ & $791 \pm 6$ & $(4.9 \pm 0.8) \%$ & $767 \pm 6$ & $(1.7 \pm 0.8) \%$ \\
$\mathrm{~B} 3$ & 751.08 & $760 \pm 6$ & $(1.2 \pm 0.8) \%$ & $784 \pm 6$ & $(4.4 \pm 0.8) \%$ & $768 \pm 6$ & $(2.2 \pm 0.8) \%$ \\
$\mathrm{C} 1$ & 759.20 & $762 \pm 6$ & $(0.4 \pm 0.8) \%$ & $788 \pm 6$ & $(3.8 \pm 0.8) \%$ & $768 \pm 6$ & $(1.2 \pm 0.8) \%$ \\
$\mathrm{C} 2$ & 754.20 & $757 \pm 5$ & $(0.4 \pm 0.7) \%$ & $781 \pm 6$ & $(3.6 \pm 0.8) \%$ & $763 \pm 5$ & $(1.2 \pm 0.7) \%$ \\
$\mathrm{C} 3$ & 746.22 & $747 \pm 5$ & $(0.1 \pm 0.7) \%$ & $747 \pm 5$ & $(0.1 \pm 0.7) \%$ & $753 \pm 5$ & $(0.9 \pm 0.7) \%$ \\
\hline
\end{tabular}

The effect of the various data sets on the translation of a reactor period to a reactivity value through the use of Eq. (1) can be summarized by looking at long reactor periods. For large values of $\tau$, Eq. (1) reduces to

$$
\rho(\tau) \stackrel{\lambda_{i} \tau \gg 1}{\longrightarrow} \frac{1}{\tau} \cdot \sum_{i=1}^{n} \frac{\beta_{i} / \beta}{\lambda_{i}} \equiv \frac{1}{\tau} \cdot R,
$$

where the proportionality constant $\mathrm{R}$ is defined as the sum of the ratios $\left(\beta_{i} / \beta\right) / \lambda_{i}$. A very simple way of comparing the delayed neutron data of various libraries is to look at the value of $R$. This value is 13.0 for the IRPhE data, the JENDL-4.0 data and the JEFF-3.3 data, while it is 11.1 for the ENDF/B-VIII.0 data. In other words, the ENDF/B-VIII.0 delayed neutron data lead to a $15 \%$ different reactivity value than the data of JEFF-3.3 and JENDL-4.0.

The benchmark value for the temperature coefficient of reactivity have been re-evaluated based on the delayed nuclear data for each library separately. The results are listed in Table V, together with updated results for the calculated values based on consistent use of nuclear data. 
Table IV. Time constants: values in the various nuclear data libraries compared with the values listed in the IRPhE benchmark description.

\begin{tabular}{|c|c|c|c|c|c|c|c|}
\hline \multirow{2}{*}{$\begin{array}{l}\text { Time } \\
\text { Group }\end{array}$} & \multicolumn{2}{|c|}{ IRPhE } & \multicolumn{2}{|c|}{ ENDF/B-VIII.0 } & \multirow{2}{*}{$\begin{array}{c}\text { JEFF-3.3 } \\
\text { All isotopes }\end{array}$} & \multicolumn{2}{|c|}{ JENDL-4.0 } \\
\hline & ${ }^{235} \mathrm{U}$ & ${ }^{238} \mathrm{U}$ & ${ }^{235} \mathrm{U}$ & ${ }^{238} \mathrm{U}$ & & ${ }^{235} \mathrm{U}$ & ${ }^{238} \mathrm{U}$ \\
\hline 1 & 0.0124 & 0.0132 & 0.013336 & 0.01363 & 0.0124667 & 0.01244 & 0.01323 \\
\hline 2 & 0.0305 & 0.0321 & 0.032739 & 0.031334 & 17 & 0.03054 & 0.03209 \\
\hline 3 & 0.111 & 0.139 & 0.12078 & 0.12334 & 5244 & 0.1114 & 0.1386 \\
\hline 4 & 0.301 & 0.358 & 0.30278 & 0.32373 & 042 & 0.3014 & 0.3591 \\
\hline 5 & 1.14 & 1.41 & 0.84949 & 0.90597 & 0.2924672 & 1.136 & 1.415 \\
\hline 6 & 3.01 & 4.02 & 2.853 & 3.0487 & 0.6664877 & 3.014 & 4.03 \\
\hline 7 & & & & & 1.634781 & & \\
\hline 8 & & & & & 3.5546 & & \\
\hline
\end{tabular}

Table V. Re-evaluated benchmark results: values based on consistent use of nuclear data.

\begin{tabular}{ccccccc}
\hline & \multicolumn{2}{c}{ ENDF/B-VIII.0 } & \multicolumn{2}{c}{ JEFF-3.3 } & \multicolumn{2}{c}{ JENDL-4.0 } \\
& Benchmark & Calculation & Benchmark & Calculation & Benchmark & Calculation \\
\hline A1a & $-1.45 \pm 0.16$ & $-1.41 \pm 0.07$ & $-1.65 \pm 0.19$ & $-1.33 \pm 0.07$ & $-1.64 \pm 0.19$ & $-1.43 \pm 0.07$ \\
A2a & $-1.07 \pm 0.09$ & $-1.18 \pm 0.07$ & $-1.23 \pm 0.11$ & $-1.17 \pm 0.07$ & $-1.23 \pm 0.11$ & $-1.19 \pm 0.07$ \\
A3 & $-1.01 \pm 0.05$ & $-0.97 \pm 0.04$ & $-1.17 \pm 0.05$ & $-0.82 \pm 0.04$ & $-1.15 \pm 0.05$ & $-0.96 \pm 0.05$ \\
\hline B1 & $-0.97 \pm 0.25$ & $-1.28 \pm 0.10$ & $-1.12 \pm 0.28$ & $-1.09 \pm 0.09$ & $-1.10 \pm 0.28$ & $-1.40 \pm 0.10$ \\
B2 & $-1.11 \pm 0.10$ & $-1.19 \pm 0.07$ & $-1.29 \pm 0.12$ & $-1.03 \pm 0.07$ & $-1.27 \pm 0.11$ & $-1.17 \pm 0.07$ \\
B3 & $-0.93 \pm 0.12$ & $-0.87 \pm 0.08$ & $-1.06 \pm 0.14$ & $-0.93 \pm 0.08$ & $-1.05 \pm 0.13$ & $-1.06 \pm 0.07$ \\
\hline C1 & $-0.70 \pm 0.03$ & $-0.57 \pm 0.06$ & $-0.80 \pm 0.03$ & $-0.53 \pm 0.05$ & $-0.80 \pm 0.03$ & $-0.74 \pm 0.05$ \\
C2 & $-0.50 \pm 0.06$ & $-0.21 \pm 0.05$ & $-0.57 \pm 0.07$ & $-0.12 \pm 0.05$ & $-0.57 \pm 0.06$ & $-0.37 \pm 0.05$ \\
C3 & $+0.13 \pm 0.03$ & $+0.43 \pm 0.05$ & $+0.15 \pm 0.03$ & $+0.47 \pm 0.04$ & $+0.15 \pm 0.03$ & $+0.46 \pm 0.05$ \\
\hline
\end{tabular}

\section{INVESTIGATION OF POSSIBLE BIAS}

The results are still not entirely satisfactory, with almost half the calculated results being more than one standard deviation away from the $1 \sigma$ experimental range. One can ask the question whether anything can be said about the validity of calculations of temperature effects by looking at critical configurations only. In this way one would avoid the influence of the delayed neutron data. In fact, the $\beta$ and $\lambda$ results reported in the previous Section were based on simulations of all critical configurations described in the benchmark. One can also use the calculated results for $k_{\text {eff, }}$ and look at trends of reactivity with temperature. The reactivity values are listed in Table VI for the three nuclear data libraries. It should be noted that in values for $k_{\text {eff, }}$ the delayed neutron data play only a minor role, whereas for $\beta_{i} / \beta$ and $\lambda_{i}$ they are dominant.

For each of the benchmark configurations, the temperature increases from sub-case 1 through sub-case 4 (A and B) or sub-case 5 (C). It can be seen in the Table that there is a trend, irrespective of the choice of nuclear data library, that the calculated reactivity is higher (or less negative) at higher temperatures. Since these models correspond to critical configurations, the ideal result would be zero for all cases, or at least more or less the same calculated reactivity value for all temperatures. In reality the calculations of reactivity at higher temperatures are higher than at lower temperatures, implying that a feedback coefficient that is based on these calculations is less negative than it should be (or too positive when the coefficient is positive as in case $\mathrm{C} 3$ ). 
Table VI. Reactivity results for each of the critical configurations in the benchmark. The temperature increases from sub-case 1 through sub-case 4 (A and B) or sub-case 5 (C).

\begin{tabular}{|c|c|c|c|c|c|c|c|c|c|}
\hline \multicolumn{2}{|c|}{$\begin{array}{c}\text { ENDF/B } \\
\text { VIII.0 }\end{array}$} & $\begin{array}{l}\text { JEFF } \\
3.3\end{array}$ & $\begin{array}{c}\text { JENDL } \\
4.0\end{array}$ & $\begin{array}{c}\text { ENDF/B } \\
\text { VIII. } 0\end{array}$ & $\begin{array}{c}\text { JEFF } \\
3.3\end{array}$ & $\begin{array}{c}\text { JENDL } \\
4.0\end{array}$ & $\begin{array}{c}\text { ENDF/B } \\
\text { VIII. } 0\end{array}$ & $\begin{array}{c}\text { JEFF } \\
3.3\end{array}$ & $\begin{array}{c}\text { JENDL } \\
4.0\end{array}$ \\
\hline & \multicolumn{3}{|c|}{ A1a } & \multicolumn{3}{|c|}{ A2a } & \multicolumn{3}{|c|}{ A3 } \\
\hline 1 & $-73 \pm 4$ & $87 \pm 4$ & $38 \pm 4$ & $-50 \pm 4$ & $102 \pm 4$ & $69 \pm 4$ & $-2 \pm 4$ & $156 \pm 3$ & $124 \pm 4$ \\
\hline 2 & $-62 \pm 4$ & $114 \pm 4$ & $52 \pm 4$ & $-30 \pm 4$ & $117 \pm 4$ & $81 \pm 4$ & $1 \pm 3$ & $119 \pm 4$ & $115 \pm 4$ \\
\hline 3 & $-28 \pm 4$ & $147 \pm 4$ & $82 \pm 4$ & $-20 \pm 4$ & $133 \pm 4$ & $91 \pm 4$ & $29 \pm 4$ & $155 \pm 3$ & $140 \pm 4$ \\
\hline \multirow[t]{2}{*}{4} & $15 \pm 4$ & $192 \pm 4$ & $125 \pm 3$ & $0 \pm 4$ & $151 \pm 4$ & $103 \pm 4$ & $38 \pm 4$ & $164 \pm 4$ & $152 \pm 4$ \\
\hline & \multicolumn{3}{|c|}{ B1 } & \multicolumn{3}{|c|}{ B2 } & \multicolumn{3}{|c|}{ B3 } \\
\hline 1 & $7 \pm 4$ & $168 \pm 4$ & $120 \pm 4$ & $-88 \pm 4$ & $85 \pm 4$ & $24 \pm 4$ & $-46 \pm 4$ & $136 \pm 4$ & $59 \pm 4$ \\
\hline 2 & $-11 \pm 4$ & $159 \pm 4$ & $96 \pm 3$ & $-87 \pm 4$ & $95 \pm 3$ & $34 \pm 4$ & $-26 \pm 4$ & $153 \pm 4$ & $53 \pm 4$ \\
\hline 3 & $3 \pm 4$ & $176 \pm 4$ & $114 \pm 4$ & $-60 \pm 4$ & $112 \pm 4$ & $48 \pm 4$ & $-14 \pm 4$ & $167 \pm 3$ & $72 \pm 4$ \\
\hline \multirow[t]{2}{*}{4} & $39 \pm 4$ & $213 \pm 3$ & $139 \pm 4$ & $-41 \pm 4$ & $133 \pm 4$ & $53 \pm 4$ & $22 \pm 3$ & $222 \pm 4$ & $119 \pm 4$ \\
\hline & \multicolumn{3}{|c|}{ C1 } & \multicolumn{3}{|c|}{$\mathrm{C} 2$} & \multicolumn{3}{|c|}{3} \\
\hline 1 & $-459 \pm 4$ & $-268 \pm 4$ & $-339 \pm 4$ & $257 \pm 4$ & $503 \pm 3$ & $379 \pm 4$ & $-263 \pm 4$ & $7 \pm 3$ & $-128 \pm 4$ \\
\hline 2 & $-447 \pm 4$ & $-251 \pm 4$ & $-322 \pm 4$ & $229 \pm 4$ & $438 \pm 4$ & $361 \pm 4$ & $-254 \pm 4$ & $12 \pm 4$ & $-125 \pm 4$ \\
\hline 3 & $-429 \pm 4$ & $-233 \pm 3$ & $-315 \pm 4$ & $245 \pm 4$ & $460 \pm 3$ & $371 \pm 4$ & $-228 \pm 4$ & $47 \pm 4$ & $-90 \pm 4$ \\
\hline 4 & $-406 \pm 3$ & $-216 \pm 4$ & $-285 \pm 4$ & $267 \pm 4$ & $485 \pm 4$ & $385 \pm 4$ & $-206 \pm 4$ & $73 \pm 3$ & $-72 \pm 4$ \\
\hline 5 & $-375 \pm 4$ & $-185 \pm 4$ & $-264 \pm 4$ & $277 \pm 4$ & $499 \pm 4$ & $404 \pm 4$ & $-154 \pm 4$ & $110 \pm 4$ & $-24 \pm 4$ \\
\hline
\end{tabular}

When one analyzes the results in Table $\mathrm{V}$, it becomes clear that the mismatch between calculations and benchmark values is in line with the trend observed in the calculations for critical configurations. As an illustration, an attempt was made to correct for the bias that is indicated by the results in Table VI. First the bias was estimated, rather crudely, by fitting the data of Table VI to a straight line around the temperature of $40^{\circ} \mathrm{C}$. The slope of this line is an estimate for the bias in the temperature coefficient of reactivity. Next, for each of the benchmark cases, the bias estimate was added to the calculated results for the temperature coefficient of reactivity. The results are shown in Figure 2. For lack of space, only the JEFF-3.3 results are shown. It appears that the presence of a bias can explain part of the discrepancies between the calculations and the benchmark values. For JENDL-4.0 (results not shown, for brevity) the results are similar. For ENDF/B-VIII.0 (results not shown, for brevity) are inconclusive, with improvements for the $\mathrm{C}$ configurations, but worse results for the $\mathrm{A}$ and $\mathrm{B}$ configurations when the bias correction is applied.

\section{CONCLUSIONS}

Calculations were performed for the TCA benchmark. Because the results were not entirely convincing, some extra work was done. First the benchmark was re-evaluated based on different delayed neutron data sets. Next it was studied whether there is a bias between calculations and benchmark, by examining only critical configurations. The conclusions are as follows.

- The TCA benchmark values depend on a choice of delayed neutron data. Implicit in the IRPhE benchmark values are the Keepin data (1965, [9]). The JEFF-3.3 and JENDL-4.0 delayed neutron data lead to almost the same benchmark values. The ENDF/B-VIII.0 delayed neutron data lead to markedly different benchmark values. 
- An analysis based on critical configurations only, avoiding the use of delayed neutron data, indicates that there is bias between calculations and benchmark. It is not clear at this point what the cause of the bias is.

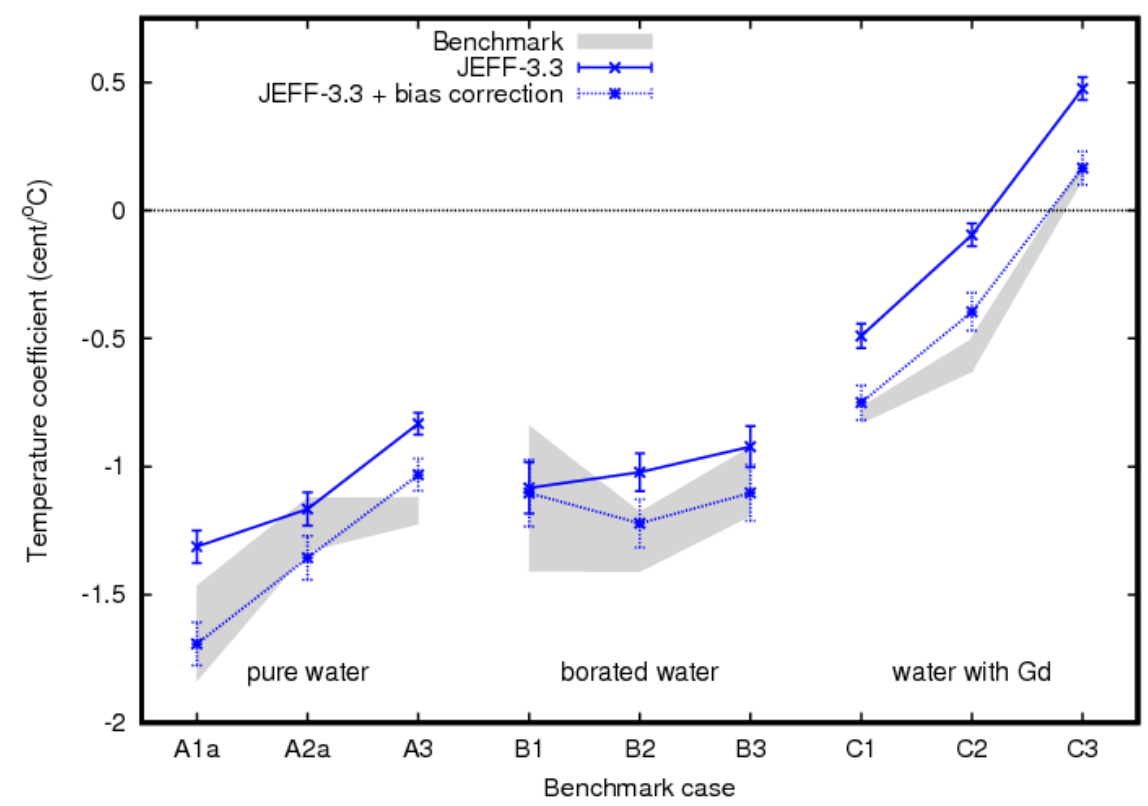

Figure 2. Results: calculated values for the temperature coefficient of reactivity, making consistent use of JEFF-3.3 nuclear data (solid lines). The results when corrected for bias are shown with dashed lines.

\section{REFERENCES}

1. "International Handbook of Evaluated Reactor Physics Benchmark Experiments (IRPhE)", Report OECD/NEA No. 7361, December 2018

2. D.B. Pelowitz, A.J. Fallgren, G.E. McMath (Eds.), "MCNP6 ${ }^{\mathrm{TM}}$ User's Manual, Code version 6.1.1beta", LA-CP-14-00745, Rev. 0 (2014)

3. D.A. Brown et al., "ENDF/B-VIII.0: The 8th Major Release of the Nuclear Reaction Data Library with CIELO-project Cross Sections, New Standards and Thermal Scattering Data", Nuclear Data Sheets 148, pp. 1-148 (2018)

4. A.J.M. Plompen et al., "The Joint Evaluated Fission and Fusion Nuclear Data Library, JEFF-3.3", submitted to Eur. J. Phys. (2019)

5. K. Shibata et al., "JENDL-4: A New Library for Nuclear Science and Engineering", J. Nucl. Sci. Technol. 48, pp. 1-30 (2011)

6. D.W. Muir, R.M. Boicort, A.C. Kahler, J.L. Conlin, W. Haeck, "The NJOY nuclear data processing system, Version 2016", LANL Report LA-UR-17-20093, 3 July 2018

7. J.I. Marquez Damian, J. R. Granada, and D. C. Malaspina, "CAB models for water: A new evaluation of the thermal neutron scattering laws for light and heavy water in ENDF-6 format", Annals of Nuclear Energy 65, pp. 280-289 (2014); private comm. J.I. Marquez Damian for the interpolator

8. A. d'Angelo, "An international cooperation to improve delayed neutron data", Progr. Nucl. En. 41, pp. 1-3 (2002), and other articles in the same issue.

9. G.R. Keepin, Physics of Nuclear Kinetics, Addison-Wesley Publ. Co., Reading, Massachusetts (1965) 